\title{
Orthopaedics in low- and middle-income countries
}

\section{Andrew Quaile ${ }^{1}$}

Published online: 5 January 2022

(c) The Author(s) under exclusive licence to SICOT aisbl 2021

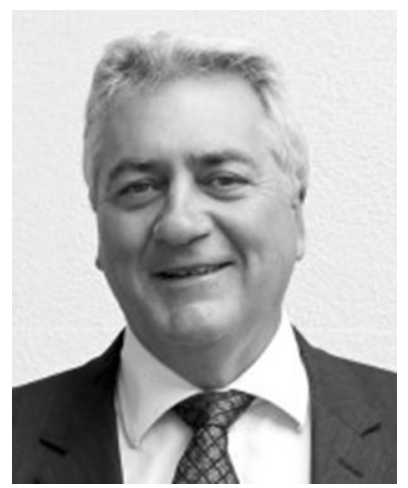

International Orthopaedics is a global orthopaedic journal that reaches throughout the world. Under its current Editorial Board and in particular leadership from its Editor, it has steadily increased its influence as a general orthopaedic journal now with an impact factor of over three. The articles published are of a very high standard which in turn creates a high impact factor; this therefore encourages excellent authors to publish in the journal. A virtuous cycle perhaps?

International Orthopaedics, unlike other journals, has a second and perhaps more important role. It is the mouthpiece of SICOT. This is the Société Internationale de Chirurgie Orthopédique et de Traumatologie which was formed in 1930 the journal being an integral part. SICOT's mission is to promote the advancement of the science and art of orthopaedics and traumatology at an international level, in particular for the improvement of patient care, and to foster and develop teaching, research and education. It maintains the philosophy that orthopaedic education should be available, accessible and appropriate for surgeons no matter their background, culture or resources. It is therefore important

Andrew Quaile

andrewquaile@icloud.com

1 Basingstoke, UK to encourage articles from areas and countries which would not normally gain much exposure.

It is, therefore, with some pride that I am able to 'Guest Edit' this special issue. It is a brave decision for the Editor and the Board to countenance an issue which may not add to the journals' journey in gaining a higher impact factor but which perhaps advances the mission of SICOT. I am hopeful that there are many articles within this issue which stimulate debate and highlight some of the problems in areas where advanced technology is not always at hand. These papers will show that despite the sort of equipment and technology we take for granted, good medicine and in particular orthopaedics can be delivered. Our colleagues that work with such limitations should be applauded and supported wherever we can. With the development of virtual clinics and advanced communications, perhaps we can avoid clinical isolation. A number of papers have discussed the support necessary to the delivery of orthopaedics particularly in relation to trauma and in development of services. Regrettably most of the impediments relate to financial support. Despite that, these papers convey a sense of hope that great things can be achieved. Papers on paediatric problems, particularly feet, are well represented along with local solutions to implant supply.

International Orthopaedics will continue to publish articles describing advanced techniques and new ideas to advance the practice of our readership. I do hope that they will continue to be a space for those articles which outline problems in the developing world, and we will continue to be the mouthpiece for SICOT. The journal would be delighted to encourage articles from beyond our 'normal' author base and would be delighted to assist with their publishing including help with any language concerns which may deter prospective authors.

Andrew Quaile

Consultant Orthopaedic and Spinal Surgeon

Deputy Editor International Orthopaedics

Basingstoke, UK

Publisher's note Springer Nature remains neutral with regard to jurisdictional claims in published maps and institutional affiliations. 